\title{
SUMAK KAWSAY Y SUMA QAMAÑA, EL RETO DE APRENDER DEL SUR. REFLEXIONES EN TORNO AL BUEN VIVIR
}

\author{
Maribel Hernández \\ Becaria FPU en el Instituto Universitario de Desarrollo Social y Paz. Universidad de Alicante
}

\section{Resumen:}

La situación actual de crisis sistémica global ha puesto de manifiesto la inviabilidad del modelo de desarrollo tradicional, basado en el crecimiento económico y la acumulación de capital como fin en sí mismo. Ante el fracaso de este modelo y la constatación de que sus dinámicas han resultado ser, en contra de lo esperado, "maldesarrolladoras", se hace necesaria la búsqueda de alternativas que propongan otras formas de relación más sostenibles y equilibradas. El ideal del Buen Vivir, originario de la cosmovisión indígena e incorporado en los nuevos textos constitucionales de Bolivia y Ecuador, promueve una nueva forma de entender el desarrollo, basada en la armonía entre los individuos y de éstos con la naturaleza. Este texto recoge las conclusiones fundamentales del seminario "Sumak Kawsay. Aprendiendo del Sur", organizado por el Instituto de Desarrollo Social y Paz de la Universidad de Alicante, precisamente con el fin de establecer espacios de discusión y análisis sobre el alcance de estas propuestas.

Palabras clave: Buen vivir, desarrollo, sumak kawsay, suma qamaña.

\begin{abstract}
The current situation of global systemic crisis has highlighted the infeasibility of the traditional development model, which is based on economic growth and capital acumulation as an end in itself. Given the failure of this model and the finding that, contrary to expectations, their dynamics are "maldevelopers", it becomes necessary to look for alternatives to propose other forms of relationship, more sustainable and balanced. The ideal of Living Well, which origin in the indigenous worldview, has been introduced
\end{abstract}


in the new constitutions of Bolivia and Ecuador. It promotes a new way of understanding development, based on harmony between individuals and between them and the nature. This text incorporates the key findings of the seminar "Sumak Kawsay. Learning from the South", organized by the Institute of Social Development and Peace at the University of Alicante, in order to provide opportunities for discussion and analysis of the scope of these proposals.

Keywords: development, sumak kawsay, suma qamaña, living well.

\section{INTRODUCCIÓN}

En un momento como en el presente, atravesado por una coyuntura económica de crisis global que ha afectado a múltiples dimensiones de la realidad social, puede resultar interesante, en la búsqueda de modelos alternativos o nuevas formas de entender la idea de desarrollo, volver la mirada al muchas veces mal llamado Sur. En América Latina, y más concretamente, en países como Ecuador o Bolivia, se vienen viviendo una serie de procesos políticos y sociales que plantean nuevas formas de relación, ya no sólo de los individuos entre sí, y de ellos con los poderes fácticos, sino del conjunto de la sociedad con la Naturaleza misma. La esencia de estos proyectos políticos emergentes se ha plasmado en un constitucionalismo de signo novedoso, articulado en torno a la idea del Buen Vivir (Sumak Kawsay, en quechua; o Suma Qamaña, en aymara), originaria de la cosmovisión indígena.

De algún modo, la coyuntura actual nos obliga a mantener una postura comprometida y a la búsqueda de propuestas que puedan ser de utilidad a la hora de abordar las cuestiones del desarrollo desde nuevas perspectivas. Con esta intención, el Instituto Universitario de Desarrollo Social y Paz de la Universidad de Alicante, reunió el pasado mes de mayo de 2009 a una serie de expertos académicos y políticos, de distintas latitudes y disciplinas, en el marco del seminario internacional "Sumak Kawsay. Aprendiendo del Sur" .

Recordando las palabras pronunciadas por el catedrático de Sociología José María Tortosa al término de su intervención en dicho seminario, ante la constatación de "una agenda que no es fácil de abordar pero ante la cual sí

1. El Seminario "Sumak Kawsay, aprendiendo del Sur" se celebró los días 28 y 29 de mayo de 2009 en la Universidad de Alicante. En él participaron: Hugo Fernández, vicecanciller de Bolivia; Koldo Unceta (UPV); José María Tortosa (UA); Leire Pajín, ex secretaria de Estado para la Cooperación Internacional; Alejandro Guillén (PYDLOS, Ecuador); Xavier Albó (CIPA, Bolivia); Alberto Acosta, ex presidente de la Asamblea Nacional Constituyente de Ecuador; Javier Arzuaga (UAEM), Juan Carlos Patiño (UAEM); o Eduardo Gudynas (CLAES, Uruguay). 
puede resultar fácil mirar hacia otro lado, lo que hemos pretendido con este seminario es aprender otras formas de acercarnos al problema para diagnosticar mejor y, en el caso de que eso sea posible, introducir las terapias oportunas". El presente artículo viene a ser una síntesis de las ideas expuestas en el transcurso de las sesiones y de las entrevistas realizadas a algunos de los ponentes invitados, en un intento de sistematizar las aportaciones que desde ámbitos como la Economía, la Sociología, la Política, la Geografía o la Ecología se vienen realizando en torno a la cuestión del Buen Vivir en las sociedades contemporáneas.

\section{PUNTO DE PARTIDA: EL MALDESARROLLO COMO DIAGNÓSTICO}

El consenso resulta unívoco. El modelo de desarrollo tradicional basado en el crecimiento económico como fin en sí mismo del desarrollo ha resultado ser inoperativo para el conjunto de la población y del planeta hasta el punto de que seguir en esta dinámica nos podría acabar conduciendo a una suerte de "suicidio colectivo", en palabras de Alberto Acosta, ex presidente de la Asamblea Nacional Constituyente de Ecuador. Esta cuestión fue ampliamente abordada por el catedrático de Economía de la Universidad del País Vasco Koldo Unceta, quien toma como punto de partida la idea de maldesarrollo de José María Tortosa. "El maldesarrollo es un concepto que se utiliza para describir el estado de cosas al que se ha llegado a través de un proceso histórico largo que, con el supuesto objetivo de incrementar el bienestar humano, ha dado como resultado un sistema generador de desigualdades, asimetrías, malestar, desequilibrios entre las personas, con la naturaleza, etc. Todo ello refleja el resultado negativo de un proceso que se suponía debía habernos llevado a un mayor bienestar, llamarlo maldesarrollo es un intento de llamar la atención sobre los aspectos negativos de ese proceso que debía conducirnos precisamente a lo contrario", sostiene Unceta.

En este sentido, Unceta habla de un "fracaso sistémico global", fracaso que se manifiesta no sólo en sus dimensiones económicas, sino también en las sociales, ecológicas o políticas. "Partíamos de una idea de progreso según la cual la expansión de la capacidad productiva crearía automáticamente el bienestar, el buen vivir", afirma. De esta noción se derivaron los conceptos de desarrollo y subdesarrollo, categorías que, hoy en día, según este investigador, "no nos ayudan a explicar el malestar actual". Parte del error ha residido en convertir los medios en fines. "El crecimiento productivo no puede ser un fin en sí mismo, sino el medio para lograr un mayor bienestar allá donde sea necesario", y un bienestar, además, que "lleve aparejada la preocupación por 
el incremento de oportunidades y capacidades de las personas". De alguna manera, para Unceta el desarrollo no es sólo una cuestión que atañe a los Estados, él rescata la idea de que las personas son fines en sí mismos del desarrollo, no sólo en tanto colectivo sino como seres individuales. "Se ha pensado que el desarrollo era cuestión de los Estados y eso, muchas veces, ha encubierto que dentro de los propios Estados había grandes desigualdades. Nunca hemos hablado de personas desarrolladas o subdesarrolladas, hablamos de países. Se daba por supuesto que en la medida en que un país se desarrollaba las personas que vivían dentro del mismo accedían directamente a un mayor bienestar. El desarrollo humano ha sido considerado un subproducto del desarrollo nacional, y éste es un tema al que habría que darle la vuelta", destaca.

Siguiendo esta línea de pensamiento, el catedrático de Sociología José María Tortosa, uno de los autores que más ha trabajado el concepto de maldesarrollo, propone partir del ideal del Buen Vivir como una vía para diagnosticar el Mal Vivir, esto es, una especie fórmula que nos permite encontrar evidencias de que el sistema mundial es maldesarrollador. Según explica, la idea de maldesarrollo "intenta referirse no a un Buen Vivir que debería buscarse sino a la constatación, primero, del fracaso del programa del "desarrollo" y, segundo, del Mal Vivir que puede observarse en el funcionamiento del sistema mundial y de sus componentes (...) Si "desarrollo" implica un elemento normativo (lo deseable), "maldesarrollo" contiene un componente empírico (lo observable) o incluso crítico (lo indeseable)". De este modo, Tortosa se sirve del conjunto de necesidades básicas (bienestar, seguridad, identidad y libertad) para diagnosticar el maldesarrollo en tres niveles: estatal/local, ecosistema y sistema mundial. Del cruce de estas variables surgen una multiplicidad de posibles indicadores del maldesarrollo, en función de la necesidad básica a la que se refieran y de su relación con cada una de estas tres escalas. Se trata de indicadores como la pobreza, la inequidad, el estancamiento económico, el calentamiento global, la polarización, marginación, violencia, terrorismo, baja calidad de la democracia, nuclearización, homogeneización, etc.

"Este contexto no es el resultado de un accidente natural", apunta Tortosa, para referirse a la existencia de una serie de "beneficiarios del maldesarrollo". "Son actores humanos que salen muy beneficiados de esta situación, que tienen su buen vivir particular", al que el investigador prefiere llamar way of life, "para no confundirlo con el otro Buen Vivir". Un way of life, cuyo máximo exponente es Estados Unidos y que se traduce, entre otros aspectos, en una "sed insaciable de materias primas en general, y de petróleo en particular", burbujas especulativas, creciente desigualdad social, gastos militares despro- 
porcionados, aumento de la deuda pública, escasa preocupación real por el medio ambiente y fórmulas de legitimación cultural. En este sentido, recuerda Tortosa, las palabras del presidente norteamericano Barak Obama, pronunciadas en el discurso inaugural de su toma de posesión, resultan ser toda una declaración de intenciones: "We will not apologize for our way of life, nor will we waver in its defense, and for those who seek to advance their aims by inducing terror and slaughtering innocents, we say to you now that our spirit is stronger and cannot be broken; you cannot outlast us, and we will defeat you"2.

\subsection{Necesidad de nuevos modelos}

El fracaso del sistema al que apuntaba Unceta, y la situación actual de maldesarrollo ponen de manifiesto la necesidad de buscar nuevos modelos capaces de superar los errores cometidos en el pasado. El reconocimiento de esta necesidad de cambio fue también el eje sobre el cual se articularon las palabras de Leire Pajín, Secretaria de Organización del Partido Socialista (PSOE). "El modelo capitalista, basado en el crecimiento económico y productivo, que conocíamos hasta hoy, tiene que cambiar. A su fracaso demostrado y reiterado en lo que se refiere a no contribuir suficientemente ni a reducir las desigualdades, ni a reducir la pobreza, ahora se suma esta crisis financiera y económica que está afectando a todos los países del planeta", sostiene.

Pajín reclama el compromiso ético de los gobiernos, que deben saber distinguir "los intereses legítimos de su país a corto plazo y saber compaginarlos con los intereses generales a largo plazo, aquellos que nos unen a los demás países y a la humanidad" como "un primer paso del Sumak Kawsay en Europa y en el mundo desarrollado". En su discurso, la que fuera Secretaria de Estado de Cooperación Internacional en España entre los años 2004 y 2008 , resalta la importancia de cambiar el modelo actual por otro que sea capaz de aprovisionar, mantener y preservar toda una serie de "bienes públicos globales" como "la calidad del medio ambiente, la salud, la integración, la estabilidad económica y social y la prevención de conflictos". Según Pajín, "si el bienestar de la población de los países llamados desarrollados está íntimamente ligado a la provisión de estos bienes públicos, esta provisión es impensable sin la colaboración, la coordinación y el compartir de todos los países del planeta", y añade que los países del Sur también tienen que tener

2. "No vamos a pedir perdón por nuestro estilo de vida, ni vamos a vacilar en su defensa, y para aquellos que pretenden lograr su fines mediante el fomento del terror y de las matanzas de inocentes, les decimos desde ahora que nuestro espíritu es más fuerte y no se puede romper; no podéis perdurar más que nosotros, y os venceremos". 
la oportunidad de "aportar y gestionar de forma compartida esos bienes públicos globales, en la medida que les corresponda, y de manera justa, para que alcancemos una respuesta suficiente entre todos". Esta necesidad de "construir un mundo más seguro y justo para todos", prosigue Pajín, "si no es por convicción, acabará siendo por egoísmo". En este sentido, afirma, "a los gobiernos del Norte no les quedará otro remedio que empezar a introducir en sus agendas estas discusiones, estas miradas alternativas, en tanto los retos de hoy, tienen repercusiones que sobrepasan los límites de nuestros propios países. No podremos abordar la inseguridad mundial, ni las consecuencias del cambio climático si no somos capaces de tejer un modelo que incluya a todos los países del planeta".

En definitiva, se trata, como concluye José María Tortosa, de cambiar las reglas del juego de un modelo que ha resultado ser madesarrollador, un sistema "basado en la eficiencia que trata de maximizar los resultados, reducir costes y conseguir la acumulación incesante de capital". Si "todo vale", afirma Tortosa, "el problema son las mismas reglas del juego dictadas y cambiadas desde arriba para satisfacer aquellos intereses". El sistema mundial, establece, "está maldesarrollado por su propia lógica y es a esa lógica a donde hay que dirigir la atención".

Este punto supone un desafío, especialmente para la ciencia económica y las metodologías que ésta ha venido utilizando tradicionalmente con la finalidad de medir y evaluar empíricamente dimensiones complejas de la realidad, como pueden ser las ideas de bienestar y desarrollo, tal y como han sido concebidas por la ciencia occidental. "Hay muchas cuestiones relacionadas con el bienestar como pueden ser la capacidad de participar, la posibilidad de tener más acceso al conocimiento, de vivir más años, de que el progreso económico sea compatible con los recursos de la naturaleza, etc., que no pueden ser evaluadas con las metodologías tradicionales de la ciencia económica", resalta Koldo Unceta. Desde Ecuador, Alberto Acosta, suscribe esta afirmación y la complementa al considerar la importancia de "repensar la Economía a partir de un par de principios básicos: la solidaridad y la reciprocidad. Esto nos lleva -afirma- a rechazar visiones estadocéntricas, pero tampoco creemos en las visiones mercadocéntricas. Creemos en una relación armónica entre Estado, mercado, y sociedad".

Precisamente, la perspectiva del Buen Vivir, y su inclusión en los nuevos textos constitucionales de Ecuador y Bolivia, marca un punto de inflexión en el debate sobre estas cuestiones y supone un paso determinante al elevar al máximo rango jurídico la noción de la armonía como uno de los valores fundamentales a seguir en la configuración de nuevas fórmulas de desarrollo. 


\section{HACIA EL BUEN VIVIR: LECCIONES DE AMÉRICA LATINA}

Un discurso común entre los participantes en el seminario fue el acuerdo en torno a la importancia de la inclusión del Buen Vivir en el texto de las nuevas constituciones de Ecuador y Bolivia, aprobadas bajo los gobiernos de Evo Morales y Rafael Correa, ya no sólo para el conjunto de sus sociedades y de cara a enriquecer el debate internacional abierto en torno a la cuestión del desarrollo, sino particularmente en lo referido a las comunidades indígenas, con quienes se venía a saldar así, de alguna manera, una deuda histórica.

Para el actual vicecanciller de Bolivia, Hugo Fernández, quien atesora una dilatada experiencia de trabajo con comunidades indígenas, la historia reciente boliviana es el resultado de un proceso, el de la emergencia del pensamiento indígena y su acceso a la política, que según explica, se inicia con la restauración de la democracia, en 1982, y que alcanza su momento más álgido con la victoria de Evo Morales, en las elecciones presidenciales de diciembre de 2005, con el 53,7 por ciento de los votos. Para Fernández, el actual presidente de Bolivia encabeza una "revolución democrática y cultural" sin precedentes en el país, a pesar de que su compleja realidad no suela ser adecuadamente retratada por parte de los medios de comunicación. El vicecanciller ilustra el alcance de esta revolución con una anécdota: "En el año 1950 los indígenas no tenían permitido el ingreso en la Plaza Murillo, que es la plaza central de la ciudad de La Paz, donde está ubicada la sede del Gobierno. Entonces, vino la Revolución de 1952, la reforma agraria de 1953, y ya se les permitió el acceso al recinto, pero no habían entrado en el Palacio Presidencial. Ahora, los indígenas han entrado en el Palacio [Presidencial] y han entrado para quedarse".

Una de las manifestaciones de esta mirada indígena incorporada a la política es, como hemos dicho previamente, la inclusión del Buen Vivir en las nuevas Constituciones. El Buen Vivir, sostiene Fernández, es "un concepto globalizante, pues así es, de algún modo, la manera de ser de los indígenas". Otro de los aspectos del Buen Vivir en el que coinciden tanto Fernández como el jesuita Xavier Albó, cofundador y primer director del Centro de Investigación y Promoción del Campesinado (CIPCA) en Bolivia, es la idea de que vivir bien no significa vivir "mejor". Según Albó, los aymaras hablan de "vivir bien, ellos no dicen vivir mejor, sino que todos vivamos bien, porque la palabra mejor resuena a que otro vive peor y el buen vivir tiene que ser algo compartido, de todos a una". Hugo Fernández añade también que no se trata de vivir mejor, "los indígenas prefieren hablar de vivir bien, de vivir en armonía, es decir, respetando y asumiendo las diferencias junto con las complementariedades. Ellos no entienden la idea de igualdad en el sentido occi- 
dental, consideran que no hay una igualdad posible, siempre hay diversidad, por eso hablan de armonía".

Si vamos un poco más allá de la semántica, podemos entender el Sumak Kawsay, siguiendo a Alejandro Guillén, director del Programa de Población y Desarrollo Local Sustentable (Pydlos) de la Universidad de Cuenca, en Ecuador, como un nuevo referente simbólico en torno al cual se han ido diseñando los derechos recogidos en estas nuevas Constituciones y sus visiones alternativas del desarrollo. Entre las bases fundamentales de este nuevo enfoque resalta la concepción de la naturaleza como sujeto de derecho, algo que se aleja transversalmente de la tradición jurídica occidental. "Dotar de derechos a la naturaleza es algo sustantivo porque rompe con la vieja tradición del pensamiento o la economía occidental, que ven a la naturaleza como un recurso. Ésta ahora pasa a ser un sujeto y un actor del desarrollo. Esto resulta fundamental porque ahora, desde la economía, ya no se trata de ver cuáles son las externalidades o los impactos ambientales, sino de cómo construir una economía que no genere dichos impactos", matiza Guillén. Dicho así, pudiera resultar incluso utópico, sin embargo, incide este investigador, es momento de "comenzar a pensar en utopías posibles". Desde su punto de vista, esa posible utopía debe pasar necesariamente "por lo humano, y ser humano no solamente en lo individual sino también en las relaciones sociales entre individuos en las comunidades, y de las comunidades contextualizadas en ambientes biofísicos con los cuales existe una relación directa".

\subsection{Una nueva visión de la naturaleza: El giro biocentrico}

Eduardo Gudynas, ecólogo social y secretario ejecutivo del CLAES (Centro Latino Americano de Ecología Social), es uno de los autores que más ha desarrollado conceptualmente la dimensión ecológica del Buen Vivir. "El Buen Vivir concibe que cualquier definición de bienestar, de calidad o de Sumak Kawsay incorpora necesariamente una dimensión ambiental, ésta ya no escapa a cualquier análisis de este tipo", resalta. Para Gudynas, esta dimensión, la incorporación de la naturaleza como sujeto de derecho en la Constitución de Ecuador, supone un auténtico cambio de paradigma, que él ha bautizado como giro biocéntrico, un cambio que conlleva importantes implicaciones sociales. "La naturaleza era objeto de derechos en tanto era objeto de uso y de apropiación por parte del ser humano. Al convertir a la naturaleza en sujeto de derecho, la Constitución ecuatoriana la está dotando de valores propios, valores intrínsecos que le son a ella misma independientemente de si es útil o económicamente utilizable para el ser humano", explica. Además, Gudynas 
recuerda que el texto constitucional plantea otra novedad radical, el derecho de restauración. "No sólo se concibe a la naturaleza como sujeto de derecho, sino que además se establece específicamente en un artículo que la restauración de los espacios naturales, de los ambientes degradados, es también un derecho de la naturaleza, que ésta tiene el derecho a ser restaurada o recuperada".

Indudablemente, este último aspecto, supone un reto importante no sólo para la Economía, sino para el resto de las Ciencias Sociales y la práctica de la gestión pública, recuerda este investigador. "La valoración económica no puede ser la regla privilegiada a la hora de tomar decisiones en las sociedades contemporáneas, hay otras dimensiones de valoración, ecológicas, religiosas, estéticas, culturales, etc., y la Economía va a tener que adaptarse a eso, tendrá que recuperar la noción de valoración múltiple, según la cual, la dimensión económica es sólo una entre varias", expone Gudynas, quien reconoce que, inevitablemente, esto hará necesario repensar el diseño de políticas públicas, "tan enfatizadas en nuestros países en la discusión de cuánto recaudo y de cuánto dinero dispongo para llevarlas a la práctica", un hecho que afecta directamente a la cuestión ambiental. Según Gudynas, "las tareas de protección ambiental, de garantía de la calidad del ambiente, o las destinadas a evitar la contaminación, hay que llevarlas a cabo independientemente de lo que cuesten". En este sentido, concluye, "el tema ambiental debería ser una política pública de responsabilidad estatal, no puede ser evaluado en términos de si es más o menos beneficioso en términos económicos".

\subsection{Caminos al Buen Vivir}

¿Y cómo llegar al Buen Vivir? Xavier Albó, siguiendo la aportación de Javier Medina en su obra "Suma Qamaña, la comprensión indígena de la Vida Buena", nos plantea una serie de posibles caminos transitables. Estos son: una sociedad de pluralidad de vida y calidad de vida; una sociedad de alta sinergia; una sociedad de baja entropía; una sociedad de equilibrio; una sociedad ecosimbiótica con su espacio; una sociedad de redes y flujos dinámicos; y una sociedad de democracias locales directas.

En suma, se trata de abogar por una construcción colectiva de la sociedad y del Buen Vivir, aspecto que entronca con la dimensión más política del Sumak Kawsay. "Es la sociedad la que construye una constitución (...), no se puede esperar entonces que la Constitución de Montecristi haga por sí sola la realidad del Buen Vivir o Sumak Kawsay", advierte Alberto Acosta, que concibe el texto constitucional como un "proyecto de vida común", "una tarea 
de ciudadanos y de ciudadanas". Tanto Acosta, como Guillén, Albó o Fernández, inciden en el aspecto relacional del Buen Vivir, en entenderlo como un proceso de constante construcción colectiva; es la idea de camino comunal o thakhi de los aymaras, quienes conciben toda la vida de cada individuo como un continuo caminar en el que la madurez se va alcanzando a medida que crece el servicio a la comunidad.

\section{A MODO DE CONCLUSIÓN}

La situación actual ha puesto de manifiesto la inviabilidad del modelo de desarrollo económico tal y como éste ha sido entendido dentro de la lógica capitalista de acumulación incesante de capital. Son muchos, y sobre distintos escenarios, los problemas que evidencian su agotamiento y la situación de crisis global a la que nos ha conducido. Sin embargo, toda crisis estructural y sistémica, como es ésta en la que nos hayamos inmersos, presenta una oportunidad ideal para poner en práctica nuevos proyectos, nuevos modelos, en definitiva, se abre la puerta a nuevas experiencias alternativas que desde los planos político, económico, ambiental y social, puedan establecer relaciones distintas fundamentadas sobre el equilibrio y la colaboración.

El momento histórico que vive América Latina, con textos constitucionales recientemente aprobados en países como Bolivia y Ecuador, que podrían ser tildados de revolucionarios, al incorporar, por primera vez, conceptos propios del pensamiento y las culturas indígenas, merece cuanto menos la atención de los países del Norte en general, y de la Academia, en particular.

El seminario "Sumak Kawsay, aprendiendo del Sur" fue concebido como un lugar de encuentro de expertos en diferentes áreas para alimentar el debate y el diálogo en torno al concepto del Buen Vivir y las implicaciones de este nuevo enfoque alrededor del cual se están reescribiendo conceptos como desarrollo o bienestar. De algún modo, el Buen Vivir plantea también un reto a las Ciencias Sociales, que están llamadas a trabajar en la elaboración de indicadores adecuados que nos ayuden a medirlo.

Merece la pena recordar aquí, para terminar, las palabras del director del Pydlos, Eduardo Guillén, respecto a las implicaciones académicas derivadas de la búsqueda de fórmulas que nos permitan medir las dimensiones del Buen Vivir, como pueden ser el respeto a los derechos de la naturaleza, los derechos comunitarios y sociales, el tema de la felicidad, el derecho al agua, a la paz... "Esto es algo que desborda a primera vista, pero ésos son los grandes retos de la Academia, y de una Academia que supere lo disciplinario y vea los problemas en su complejidad a través de la interdisciplinaridad (...) Las Ciencias 
Sociales deben ser capaces de explicar los procesos de la vida, los procesos sociales, comunitarios, y su relación con un ambiente que tiene sus propios derechos como ser vivo. Todo eso es sumamente complicado, pero si no apuestas, si no tienes utopías, acabas cayendo en el tecnicismo. El tecnicismo te puede dar soluciones a lo concreto, a lo inmediato, pero no da soluciones a tu vida como ser humano. Y al final, lo esencial, es el concepto de vida". 